A C G

publications

Rec. Nat. Prod. 14:1 (2020) 83-88

records of natural

products

\title{
Natural Glycosides from Indigofera stachyoides radix
}

\author{
Wei Zhou $\odot^{1 \#,}$ Zhong Lei $\odot^{1,2 \#, ~ D o n n a ~ L a i ~} \odot^{3}$, George Q. Li $\odot^{3}$, \\ Yan Liang $\odot^{1}$, Xiaoyan Zhang $\odot^{2}$, Junlae Cho $\odot^{3^{*}}$ and Xiaoyan Hao $\odot^{1^{*}}$ \\ ${ }^{1}$ School of Pharmacy, Guizhou Medical University, Guiyang 550025, Guizhou, China \\ ${ }^{2}$ School of Basic Medical Sciences, Guizhou Medical University, Guiyang 550025, Guizhou, China \\ ${ }^{3}$ Faculty of Medicine and Life Science, The University of Sydney, NSW, 2006, Australia
}

(Received April 08, 2019; Revised June 24, 2019; Accepted June 25, 2019)

\begin{abstract}
Six natural polar glycosides were isolated and identified from indigofera stachyoides radix, they were identified as $\beta$-sitosterol-D-glucoside (1), schizandriside (2), kaempferol-7-O- $\beta$-D-glucopyranoside (3), 3,4,5trimethoxyphenyl-O- $\beta$-D-glucopyranoside (4), 2-methoxy-4-(2'-hydroxyethyl)-phenol-1-O- $\beta$-D-glucopyrano-side (5) and 2-(3-hydroxy-4-methoxyphenyl) ethyl 1-O- $\beta$-D-glucopyranoside (6). Meanwhile, four flavonoids including 7, 4'dihydroxyl-3'-methoxyisoflavone (7), calycosin (8), 7-hydroxyl-4'-methoxyflavanone (9), maackiain (10) and one steroid compound, stigmasterol (11) were also reported herein.
\end{abstract}

Key words: Indigofera stachyoides radix; glycoside; schizandriside; kaempferol-7-O- $\beta$-D-glucopyranoside, natural medicine. (C) 2019 ACG Publications. All rights reserved.

\section{Plant Source}

Indigofera stachyoides radix, the dry roots of Indigofera stachyoides Lindl. were collected in May 2016 from Liuzhi Special District of Guizhou Province, the southwest China, and were authenticated by Professor Deyuan Chen, Guiyang College of Traditional Chinese Medicine. A sample (No. 201605A) was deposited in Herbarium of Chinese Materia Medica and Ethnomedicines, School of Pharmacy, Guizhou Medical University.

\section{Previous Studies}

Indigofera stachyoides radix, named Xuerenshen in pinyin as Chinese medicine in the southwest China has been clinically proven to stimulating blood circulation, eliminating dampness \& phlegm and relieving exterior syndrome based on traditional Chinese medicine theory (TCM). It has been used for treatment of cold, fever, headache, ulcer, women's abdominal pain \& massive vaginal bleeding [1,2]. Indigofera stachyoides radix as the assistant herbal medicine in Qijiao Shengbai Capsule $\square$ a kind of Chinese proprietary medicines plays an important therapeutic role. Our group has carried out systematic research on its medicinal substances, found its hepatoprotective activity [3-6]. In the actual research process, most compounds in this

\footnotetext{
"Corresponding author: junlae.cho@sydney.edu.au 2604084238@qq.com Phone: 086-851-88416165

\# These authors contributed equally to this work
} 


\section{Glycosides from Indigofera stachyoides radix}

medicinal plant showed relatively large polarity and even unstable. In this work, we further had enriched chemical constituents of Indigofera stachyoides radix to provide a clear material basis for its new drug development and modernization of TCM.

\section{Present Study}

The dry root of Indigofera stachyoides Lindl. $20.0 \mathrm{~kg}$ were pulverized to crude powder ( $80 \mathrm{mesh}$ ), then extracted three times with $95 \%$ ethanol under reflux $(4 \mathrm{~h}, 4 \mathrm{~h}, 3 \mathrm{~h})$. After the extraction solution was filtered, the residue was further under reflux extraction using $50 \%$ ethanol for $2 \mathrm{~h}$. All filtration solution was merged, concentrated under reduced pressure to obtain the crude extract which was suspended in water and partitioned successively with petroleum ether, ethyl acetate, and n-butyl alcohol, respectively. After evaporation of the solvent under reduced pressure, the ethyl acetate extract $496 \mathrm{~g}$ was obtained, then subjected to silica-gel (200-300 mesh, Qingdao Marine Chemical, China) column chromatography with chloroformmethanol (19:1 1:1) as the gradient eluent to yield five fractions (Fr1 Fr5).

The Fr1 (12.1 g) was chromatographed on a silica gel column using a gradient solvent petroleum etheracetone (200:1 1:1) to give seven subfractions (Fr1-1 Fr1-7). Compound 11 (100.0 mg) was obtained by recrystallization from Fr1-2. Fr1-3 was further purified by LH-20 column (GE Healthcare, Sweden) eluted with acetone to obtain subfractions, compound $\mathbf{6}(9.0 \mathrm{mg})$ and compound $\mathbf{1 0}(25.0 \mathrm{mg})$ were afforded by silica gel column chromatography with petroleum ether-ethyl acetate $(20: 1,10: 1)$. Fr1-4 was purified by LH20 column eluted with dichloromethane-methanol (1:1), the subfractions were subjected to HPLC C18 column chromatography using the mobile phase of acetonitrile : water (23:77) to get compounds 7 ( $2.3 \mathrm{mg}$ ), $8(3.0 \mathrm{mg})$ and $9(2.5 \mathrm{mg})$. Fr4 (145 g) was chromatographed on MCI gel column (75-150 $\mu \mathrm{m}$, Mitsubishi Chemical, Japan) eluting with methanol : water (5:95) to afford (Fr4.1 Fr4-5). Fr4-1 was further purified by LH-20 column eluted with methanol : water (1:1), and then Semi-preparative HPLC C18 column chromatography (Welch Ultimate XB-C18, $4.6 \mathrm{~mm} \times 250 \mathrm{~mm}, 10 \mu \mathrm{m}$ ) using the mobile phase of acetonitrile : water (7:93) to get compounds $4(8.0 \mathrm{mg})$ and $\mathbf{5}(9.0 \mathrm{mg})$. Compound $\mathbf{1}(50.0 \mathrm{mg})$ was obtained by recrystallization from Fr4-2. Fr4-4 was purified to afford compound $\mathbf{3}(10 \mathrm{mg})$ by LH-20 column eluted with methanol, the eluted residue was finally recrystallized to get compound 2 (200 $\mathrm{mg})$. All NMR spectra were recorded on Bruker AV-600 NMR, DRX-500 NMR spectrometers. ESI-MS spectra were recorded on Shimadzu LC-IT-TOF, Thermo TSQ Endura LC/MS/MS.

Compound 1 was obtained as a white powder. ${ }^{1} \mathrm{H}$ NMR $(500 \mathrm{MHz}, \mathrm{DMSO}-d 6) \delta: 5.32(1 \mathrm{H}, \mathrm{d}, J=4.8$ Hz, H-1'), 4.90 (3H, m, H-6, 4'-OH, 2'-OH), 4.46 (1H, t, $\left.J=5.8 \mathrm{~Hz}, 3^{\prime}-\mathrm{OH}\right), 4.21$ (1H, d, $\left.J=7.8 \mathrm{~Hz}, 66^{\prime}-\mathrm{OH}\right)$, $3.64\left(1 \mathrm{H}, \mathrm{dd}, J=11.4,7.2 \mathrm{~Hz}, \mathrm{H}-2^{\prime}\right), 3.09\left(5 \mathrm{H}, \mathrm{m}, \mathrm{H}-3^{\prime}, 4^{\prime}, 5^{\prime}, 6^{\prime}, 6^{\prime}\right), 2.89(1 \mathrm{H}, \mathrm{td}, J=8.6,4.8 \mathrm{~Hz}, \mathrm{H}-3), 2.36$ $(1 \mathrm{H}, \mathrm{dd}, J=12.4,3.5 \mathrm{~Hz}, \mathrm{H}-4), 2.12(1 \mathrm{H}, \mathrm{t}, J=12.3 \mathrm{~Hz}, \mathrm{H}-7), 1.93(2 \mathrm{H}, \mathrm{m}, \mathrm{H}-4,7), 1.79(2 \mathrm{H}, \mathrm{m}, \mathrm{H}-15$, 16), 1.51 (7H, m, H-2, 11, 12, 15, 16, 28, 28), $1.27-0.98$ (16H, m, H-1, 1, 2, 8, 9, 11, 12, 14, 17, 20, 22, 22, 23, 23, 24, 25), $0.81\left(18 \mathrm{H}, \mathrm{m}, 18,19,21,26,27,29-\mathrm{CH}_{3}\right) .{ }^{13} \mathrm{C}$ NMR (126 MHz, DMSO-d6) $\delta: 140.51$ (C-5), 121.31 (C-6), 100.83 (C-1'), 76.99 (C-5'), 76.80 (2C-3, 3'), 73.52 (C-2'), 70.14 (C-4'), 61.14 (C-6'), 56.25 (C14), 55.48 (C-17), 49.67 (C-9), 45.19 (C-24), 41.92 (C-4), 41.81 (C-13), 38.36 (C-12), 36.90 (C-1), 36.28 (C-10), 35.56 (C-20), 33.40 (C-22), 31.48 (C-8), 31.44 (C-7), 29.32 (C-2), 28.75 (C-25), 27.88 (C-23), 25.46 (C-15), 23.94 (C-16), 22.66 (C-28), 20.67 (C-11), 19.80 (C-27), 19.18 (C-26), 19.00 (C-21), 18.69 (C-19), 11.85 (C-29), 11.74 (C-18). The above NMR data was consistent with the reference [7], this compound was eventually identified as $\beta$-sitosterol-D-glucoside (RN 474-58-8) (Figure 1).

Compound 2 was obtained as white needle-like crystals. Its ESI-MS peak at $\mathrm{m} / z 491.16[\mathrm{M}-\mathrm{H}]^{-} \cdot{ }^{1} \mathrm{H}$ NMR (500 MHz, CD 30 OD) $\delta: 6.73\left(1 \mathrm{H}, \mathrm{d}, J=2.0 \mathrm{~Hz}, \mathrm{H}-2^{\prime}\right), 6.69\left(1 \mathrm{H}, \mathrm{d}, J=8.1 \mathrm{~Hz}, \mathrm{H}-5^{\prime}\right), 6.60(1 \mathrm{H}, \mathrm{s}, \mathrm{H}-$ 2), $6.58\left(1 \mathrm{H}, \mathrm{dd}, J=8.1,2.0 \mathrm{~Hz}, \mathrm{H}-6{ }^{\prime}\right), 6.12(1 \mathrm{H}, \mathrm{s}, \mathrm{H}-5), 4.01(2 \mathrm{H}, \mathrm{m}, \mathrm{H}-9), 3.93(1 \mathrm{H}, \mathrm{dd}, J=9.8,2.4 \mathrm{~Hz}$, H-1"), $3.77\left(1 \mathrm{H}, \mathrm{d}, J=5.6 \mathrm{~Hz}, \mathrm{H}-7^{\prime}\right), 3.76\left(3 \mathrm{H}, \mathrm{s}, 3^{\prime}-\mathrm{OCH}_{3}\right), 3.75\left(3 \mathrm{H}, \mathrm{s}, 3-\mathrm{OCH}_{3}\right), 3.67$ (2H, m, H-9'), 3.41 $\left(2 \mathrm{H}, \mathrm{m}, \mathrm{H}-5^{\prime \prime}\right), 3.16$ (2H, m, H-2", 3"), 3.07 (1H, m, H-4"), 2.77 (2H, m, H-7), $2.02\left(1 \mathrm{H}, \mathrm{m}, \mathrm{H}-8^{\prime}\right), 1.80$ (1H, $\mathrm{t}, J=10.4 \mathrm{~Hz}, \mathrm{H}-8) .{ }^{13} \mathrm{C}$ NMR (126 MHz, CD $\mathrm{CDD}_{3} \mathrm{O} \delta: 148.92$ (C-3'), 147.15 (C-3), 145.83 (C-4'), 145.13 (C4), 138.63 (C-1'), 134.31 (C-6), 129.10 (C-1), 123.11 (C-6'), 117.37 (C-5), 116.07 (C-5'), 114.23 (C-2'), 112.35 (C-2), 105.82 (C-1"), 77.91 (C-3"), 75.00 (C-2"), 71.26 (C-4"), 69.38 (C-9'), 66.91 (C-5"), 65.09 (C9), $56.43\left(3^{\prime}-\mathrm{OCH}_{3}\right), 56.36\left(3-\mathrm{OCH}_{3}\right), 49.85,47.92\left(\mathrm{C}-7^{\prime}\right), 45.87(\mathrm{C}-8$ '), $39.58(\mathrm{C}-8), 33.85(\mathrm{C}-7)$. The above NMR data was consistent with the reference [8], this compound was eventually identified as schizandriside (RN 80734-72-1). 
Zhou et al., Rec. Nat. Prod. (2020) 14:1 83-88

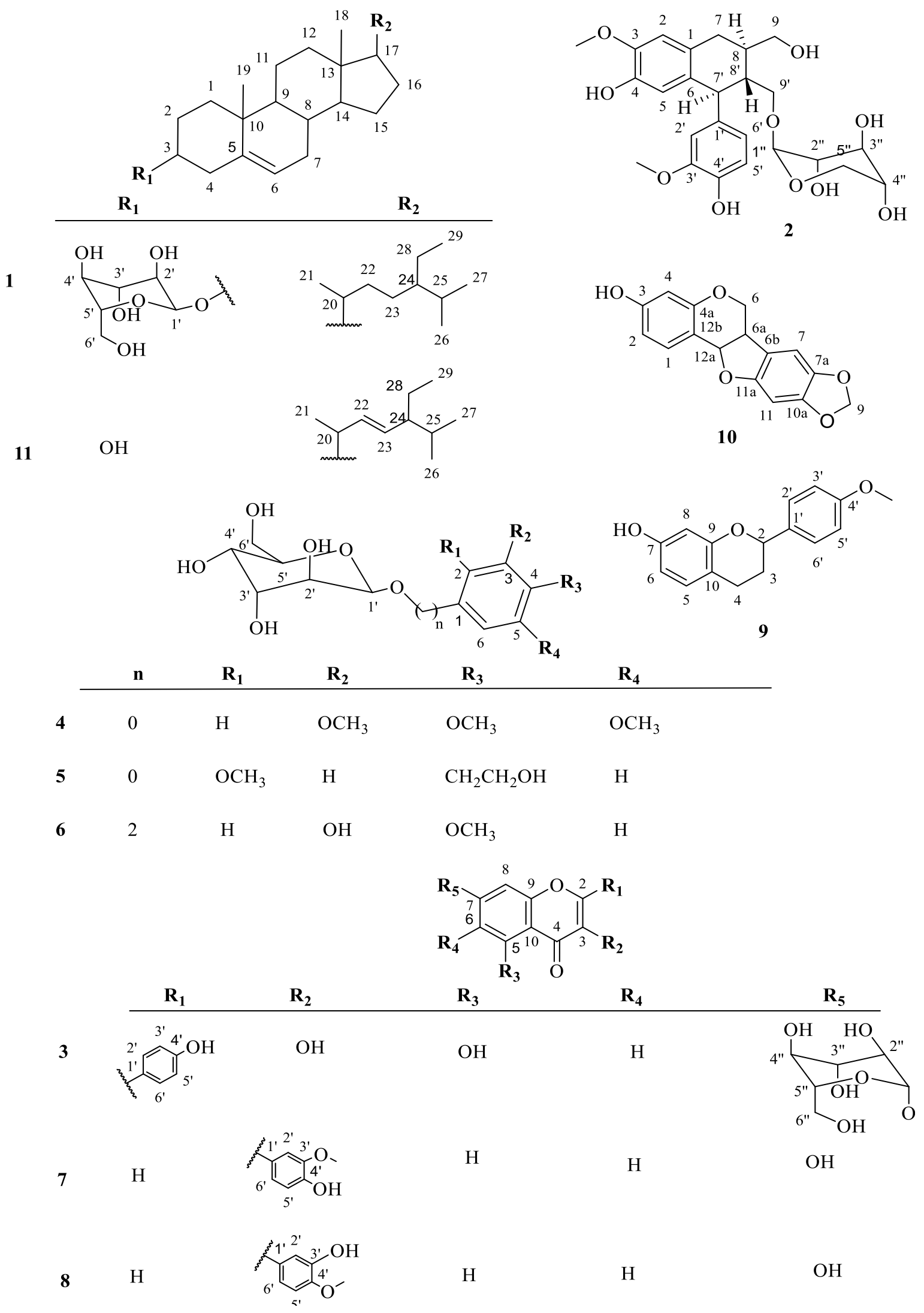

Figure 1. Structures of compounds 1-11 
Compound 3 was yellow needle-like crystals. Its ESI-MS peak at $m / z 446.99[\mathrm{M}-\mathrm{H}]^{-}{ }^{-}{ }^{1} \mathrm{H}$ NMR $(600$ $\left.\mathrm{MHz}, \mathrm{CD}_{3} \mathrm{OD}\right) \delta: 8.12\left(2 \mathrm{H}, \mathrm{d}, J=8.4 \mathrm{~Hz}, \mathrm{H}-2^{\prime}, 6^{\prime}\right), 6.90(2 \mathrm{H}, \mathrm{d}, J=8.4 \mathrm{~Hz}, \mathrm{H}-3$ ', 5'), $6.76(1 \mathrm{H}, \mathrm{s}, \mathrm{H}-8), 6.45$ $(1 \mathrm{H}, \mathrm{d}, J=2.2 \mathrm{~Hz}, \mathrm{H}-6), 5.05(1 \mathrm{H}, \mathrm{d}, J=6.8 \mathrm{~Hz}, \mathrm{H}-1 "), 3.93(1 \mathrm{H}, \mathrm{dd}, J=12.2,2.3 \mathrm{~Hz}, \mathrm{H}-2 "), 3.71(1 \mathrm{H}, \mathrm{dd}$, $J=12.1,5.9 \mathrm{~Hz}, \mathrm{H}-3 "), 3.54$ (1H, ddd, $J=8.4,5.9,2.2 \mathrm{~Hz}, \mathrm{H}-5 "), 3.48$ (2H, m, H-4", 6"), 3.40 (1H, t, $J=$ $8.9 \mathrm{~Hz}, \mathrm{H}-6 ") .{ }^{13} \mathrm{C}$ NMR (151 MHz, CD $\left.\mathrm{OD}\right) \delta: 177.58$ (C-4), 164.48 (C-7), 162.19 (C-5), 160.76 (C-4'), 157.76 (C-9), 148.81 (C-2), 137.61 (C-3), 130.89 (2C-2', 6'), 123.57 (C-1'), 116.33 (2C-3', 5'),

106.31 (C-10), 101.64 (C-1"), 100.23 (C-6), 95.57 (C-8), 78.37 (C-5"), 77.85 (C-3"), 74.75 (C-2"), 71.28 (C4"), 62.46 (C-6"). The above NMR data was consistent with the reference [9], this compound was eventually identified as kaempferol-7-O- $\beta$-D-glucopyranoside (RN 16290-07-6).

Compound 4 was white powder, its NMR data in Table 1 was consistent with the reference[10], identified as 3,4,5-trimethoxyphenyl-O- $\beta$-D-glucopyranoside (RN 41514-64-1). Compound 5 was white powder, inferred as 2-methoxy-4-(2'-hydroxyethyl)-phenol-1-O- $\beta$-D-glucopyranoside[11]. Compound $\mathbf{6}$ was white powder, the ESI-MS peak at $\mathrm{m} / z 353[\mathrm{M}+\mathrm{Na}]^{+}$, 2-(3-hydroxy-4-methoxyphenyl) ethyl 1-O- $\beta$-Dglucopyranoside (RN 125180-72-5) was finally confirmed [12].

Table 1. ${ }^{1} \mathrm{H}$ NMR $(600 \mathrm{MHz})$ and ${ }^{13} \mathrm{C}$ NMR $(151 \mathrm{MHz})$ data of compound s 4-6 in $\mathrm{CH}_{3} \mathrm{OD}(\delta$ in ppm and $J$ in $\mathrm{Hz})$

\begin{tabular}{|c|c|c|c|c|c|c|}
\hline \multirow[t]{2}{*}{ Position } & \multicolumn{2}{|l|}{4} & \multicolumn{2}{|l|}{5} & \multicolumn{2}{|l|}{6} \\
\hline & $\delta_{\mathrm{H}}$ & $\delta_{\mathrm{C}}$ & $\delta_{\mathrm{H}}$ & $\delta_{\mathrm{C}}$ & $\delta_{\mathrm{H}}$ & $\delta_{\mathrm{C}}$ \\
\hline 1 & - & 154.78 & - & 146.42 & - & 131.56 \\
\hline 2 & $6.48(\mathrm{~s})$ & 96.03 & & 150.69 & $6.85(\mathrm{~d} 2.0)$ & 116.04 \\
\hline 3 & - & 154.78 & $6.88(\mathrm{~d} 2.0)$ & 114.55 & - & 148.81 \\
\hline 4 & - & 134.36 & & 135.48 & - & 145.90 \\
\hline 5 & - & 156.07 & $6.76(\mathrm{dd} 8.2,2.0)$ & 122.51 & $6.68(\mathrm{~m})$ & 113.71 \\
\hline 6 & $6.48(\mathrm{~s})$ & 96.03 & $7.08(\mathrm{~d} 8.2)$ & 118.26 & $6.68(\mathrm{~m})$ & 122.38 \\
\hline 7 & - & - & $3.72(\mathrm{t} 7.0)$ & 64.27 & $2.84(\operatorname{td} 7.3,2.7)$ & 71.99 \\
\hline 8 & - & - & $2.76(\mathrm{t} 7.0)$ & 39.81 & $\begin{array}{l}3.86(\text { dd } 11.9,2.7) \\
3.65(\text { dd } 11.9,5.3)\end{array}$ & 36.75 \\
\hline 9 & - & - & - & - & - & - \\
\hline 10 & - & - & - & - & - & - \\
\hline $1^{\prime}$ & $4.80(\mathrm{~d} 7.5)$ & 103.18 & $4.83(\mathrm{~d} 7.4)$ & 103.07 & $4.29(\mathrm{~d} 7.8)$ & 104.32 \\
\hline $2^{\prime}$ & $4.80(\mathrm{~d} 7.5)$ & 74.93 & $4.83(\mathrm{~d} 7.4)$ & 74.93 & $4.05(\mathrm{~m})$ & 75.12 \\
\hline $3^{\prime}$ & $3.42(\mathrm{~m})$ & 78.06 & $3.68(\mathrm{dd} 12.0,5.1)$ & 77.82 & $3.71(\mathrm{~m})$ & 78.09 \\
\hline $4^{\prime}$ & $3.42(\mathrm{~m})$ & 71.69 & $3.46(\mathrm{~m})$ & 71.33 & $3.26(\mathrm{~m})$ & 71.64 \\
\hline $5^{\prime}$ & $3.42(\mathrm{~m})$ & 78.43 & $3.46(\mathrm{~m})$ & 78.17 & $3.26(\mathrm{~m})$ & 77.95 \\
\hline $6^{\prime}$ & $\begin{array}{l}3.91(\mathrm{dd} 12.0,2.2) \\
3.65(\mathrm{dd} 12.0,6.7)\end{array}$ & 62.71 & $3.37(\mathrm{dd} 6.5,1.6)$ & 62.49 & $\begin{array}{l}3.34(\mathrm{t} 9.1), 3.18 \\
(\mathrm{dd} 9.1,8.0)\end{array}$ & 62.75 \\
\hline $2-\mathrm{OCH}_{3}$ & - & - & $3.85(\mathrm{~s})$ & 56.67 & - & - \\
\hline $3-\mathrm{OCH}_{3}$ & $3.80(\mathrm{~s})$ & $56.51(\mathrm{~s})$ & - & - & - & - \\
\hline $4-\mathrm{OCH}_{3}$ & $3.69(\mathrm{~s})$ & $61.21(\mathrm{~s})$ & - & - & $3.83(\mathrm{~s})$ & 56.35 \\
\hline $5-\mathrm{OCH}_{3}$ & $3.80(\mathrm{~s})$ & $56.51(\mathrm{~s})$ & - & - & - & - \\
\hline
\end{tabular}

Compound 7 was pale yellow powder. Its ESI-MS peak at $\mathrm{m} / z$ 283.0611 [M-H] $]^{-}$. The location of all carbons was finally determined by DEPT90 and DEPT135 data of this compound. The ${ }^{1} \mathrm{H}$ NMR spectrum of compound 7 displayed four ortho-coupling aromatic protons at $\delta_{\mathrm{H}} 7.96,6.82(1 \mathrm{H} \mathrm{each}, \mathrm{d}, 8.9 \mathrm{~Hz})$ and $\delta_{\mathrm{H}}$ 6.94, $6.83(1 \mathrm{H}$ each, d, $8.2 \mathrm{~Hz})$, two intersite-coupling aromatic protons at $\delta_{\mathrm{H}} 7.15(1 \mathrm{H}, \mathrm{d}, 2.0 \mathrm{~Hz})$ and $\delta_{\mathrm{H}}$ $6.67(1 \mathrm{H}, \mathrm{d}, 1.7 \mathrm{~Hz})$ (Table 2). The HSQC spectrum showed that there were two COSY correlations of C-H. The HMBC cross-peaks from $\delta_{\mathrm{H}} 8.08(\mathrm{H}-2)$ to C-3, C-4 and C-9, from $\delta_{\mathrm{H}} 7.96(\mathrm{H}-5)$ to C-4, C-7 and C-9 together with the COSY correlation of $\mathrm{H}-5 / \mathrm{H}-6 / \mathrm{H}-8$ indicated the assignment of these protons at $\mathrm{C}-2$ and $\mathrm{C}$ 5 respectively. Besides, a methoxy group was observed at $\delta_{\mathrm{H}} 3.88\left(3 \mathrm{H}, \mathrm{s}, 3^{\prime}-\mathrm{OCH}_{3}\right)$ corresponding to the carbon signal at $\delta_{\mathrm{C}} 56.41$ in the HMBC spectrum (Figure S31 in supporting information). The HMBC correlation from $\delta_{\mathrm{H}} 3.88\left(3^{\prime}-\mathrm{OCH}_{3}\right)$ to $\delta_{\mathrm{C}} 148.76$ demonstrated this methoxy group linked to C-3'. These above evidence revealed an isoflavone unit in the structure of compound 7 , the compound was eventually identified as a natural compound named 7, 4'-dihydroxyl-3'-methoxyisoflavone. Compound $\mathbf{8}$ was pale 
Zhou et al., Rec. Nat. Prod. (2020) 14:1 83-88

yellow powder. Its ESI-MS peak at $m / z 283[\mathrm{M}-\mathrm{H}]^{-}$, its structure was found to be similar with compound 7 except chemical substituents of C-3' and C-4', the NMR data was consistent with the reference [13], it was identified as calycosin (RN 20575-57-9). Compound 9 was pale yellow powder, this compound was eventually identified as 7-hydroxyl-4'-methoxyflavanone (RN 108837-20-3) whose NMR data was consistent with the reference [14]. Compounds 10, 11 were maackiain (RN 2035-15-6) [15] and stigmasterol (RN 83-48-7) [16,17], the detailed spectral data were listed in supporting information of this article.

In this study, The compounds 4, 5, 6 and 9 were isolated from the family Leguminosae for the first time, the compounds $\mathbf{3}, \mathbf{7}$ and $\mathbf{8}$ were first reported from the genus Indigofera.

Table 2. ${ }^{1} \mathrm{H}$ NMR $(600 \mathrm{MHz})$ and ${ }^{13} \mathrm{C}$ NMR $(151 \mathrm{MHz})$ data of compounds 7-9 in $\mathrm{CH}_{3} \mathrm{OD}(\delta$ in ppm and $J$ in $\mathrm{Hz})$

\begin{tabular}{|c|c|c|c|c|c|c|}
\hline \multirow[t]{2}{*}{ Position } & \multicolumn{2}{|l|}{7} & \multicolumn{2}{|l|}{8} & \multicolumn{2}{|l|}{9} \\
\hline & $\delta_{\mathrm{H}}$ & $\delta_{\mathrm{C}}$ & $\delta_{\mathrm{H}}$ & $\delta_{\mathrm{C}}$ & $\delta_{\mathrm{H}}$ & $\delta_{\mathrm{C}}$ \\
\hline 2 & $8.08(\mathrm{~s})$ & 154.38 & $8.11(1 \mathrm{H}, \mathrm{s})$ & 154.75 & $5.37(\mathrm{dd} 13.2,2.9)$ & 81.04 \\
\hline 3 & - & 125.59 & - & 125.72 & $\begin{array}{c}3.04(\mathrm{dd} 17.0,13.2), \\
2.68(\mathrm{dd} 17.0,2.9)\end{array}$ & 44.96 \\
\hline 4 & - & 178.16 & - & 178.03 & - & 193.53 \\
\hline 5 & $7.96(\mathrm{~d} 8.9)$ & 127.94 & $8.02(\mathrm{~d} 8.8)$ & 128.39 & $7.72(\mathrm{~d} 8.8)$ & 129.85 \\
\hline 6 & $6.82(\mathrm{dd} 8.9,1.7)$ & 118.96 & $6.90(\mathrm{dd} 8.8,2.3)$ & 117.04 & $6.49(\mathrm{dd} 8.8,2.3)$ & 111.83 \\
\hline 7 & - & 169.82 & - & 165.90 & - & 167.01 \\
\hline 8 & 6.67 (d 1.7) & 103.71 & $6.80(\mathrm{~d} 2.3)$ & 103.35 & $6.34(\mathrm{~d} 2.3)$ & 103.84 \\
\hline 9 & - & 160.52 & - & 159.93 & - & 165.60 \\
\hline 10 & - & 116.01 & - & 117.70 & - & 114.90 \\
\hline $1^{\prime}$ & - & 125.19 & - & 126.31 & - & 131.37 \\
\hline $2^{\prime}$ & $7.15(\mathrm{~d} 2.0)$ & 114.15 & 7.03 (br s) & 117.41 & $7.32(\mathrm{~d} 8.6)$ & 129.01 \\
\hline $3^{\prime}$ & - & 148.76 & - & 147.42 & $6.81(\mathrm{~d} 8.6)$ & 116.30 \\
\hline 4' & - & 147.76 & - & 149.14 & - & 158.98 \\
\hline $5^{\prime}$ & $6.83(\mathrm{~d} 8.2)$ & 116.14 & 6.96 (br s) & 112.60 & $6.81(\mathrm{~d} 8.6)$ & 116.30 \\
\hline $6^{\prime}$ & $6.94(\mathrm{dd} 8.2,2.0)$ & 122.88 & 6.96 (br s) & 121.60 & $7.32(\mathrm{~d} 8.6)$ & 129.01 \\
\hline $6-\mathrm{OH}$ & - & - & - & - & - & - \\
\hline 3'-OH & - & - & - & - & - & - \\
\hline $4^{\prime}-\mathrm{OCH}_{3}$ & $3.88(\mathrm{~s})$ & 56.41 & $3.87(\mathrm{~s})$ & 56.40 & $3.87(\mathrm{~s})$ & 56.45 \\
\hline
\end{tabular}

\section{Acknowledgments}

This work was financially supported by National Natural Science Foundation of China (81460640, 81360681), Guizhou Provincial High-Level Innovative Talents Project "Thousand-Level" (No. 2016-2018) and Scholarship of China Scholarship Council (No. 201408525053).

\section{Supporting Information} products

Supporting information accompanies this paper on http://www.acgpubs.org/journal/records-ofnatural-

\section{ORCID}

Wei Zhou:0000-0002-4123-2276

Zhong Lei:0000-0002-1779-8755

Donna Lai:0000-0002-6593-3149

George Q. Li:0000-0002-9633-865X

Yan Liang:0000-0002-3458-6818

Xiaoyan Zhang: 0000-0002-1597-3038

Junlae Cho:0000-0002-0540-3234

Xiaoyan Hao:0000-0002-4370-8821 
Glycosides from Indigofera stachyoides radix

\section{References}

[1] Guizhou Provincial Drug Administration (2003). Quality Standards of Traditional Chinese Medicines and Ethnic Medicines in Guizhou Province (2003 edition). Guiyang: Guizhou Science and Technology Press, p169.

[2] X. G. Wang and R. G. Zhang (2015). Research progress of Radix Indigofera, Chin. J. Ethnomed. Ethnopharm. 24, 21-22.

[3] L. Qiu, Y. Liang, G. H. Tang, C. M. Yuan, Y. Zhang, X. Y. Hao, X. J. Hao and H. P. He (2013). Two new flavonols, including one flavan dimer, from the roots of Indigofera stachyodes, Phytochem. Lett. 6, 368-371.

[4] L. Qiu, Y. Liang, G. H. Tang, C. M. Yuan, X. Y. Hao and H. P. He (2013). Chemical constituents from the roots of Indigofera stachyoides, Chin Tradit Patent Med. 35, 320-323.

[5] H. Yang, Y. S. Yang, F. Peng, J. Liu, Q. Wu, X. Y. Hao and J. Z. Shi (2018). Effects of ethyl acetate extracts from Indigofera stachyoides Radix against carbon tetrachloride induced liver injury in mice, Lishizhen Med. Materia Medica Res. 29, 2354-2357.

[6] Z. Lei, X. Y. Zhu, Y. S. Yang, W. Zhou, Y. Liang and X. Y. Hao (2018). Hepatoprotective chemical constituents from ethylacetate extract of Indigofera stachyoides Radix, Chin. J. Exper. Tradit. Med. Formulae 24, 56-63.

[7] L. M. Li, W. L. Li, Q. S. Guo, B. R. Ren and H. Q. Zhang (2008). Studies on the chemical constituents from Gynura divaricata (L.) DC, Lishizhen Med. Materia Medica Res. 19(1), 118-119.

[8] J. F. Xu, F. S. Li, Z. M Feng, J. S. Jiang and P. C. Zhang (2011). A new sesquiterpenoid from mallotus apelta, Chem. Nat. Comp. 47(2), 218-219.

[9] D. Guo, W. J. Xue, G. A. Zou and H. A. Aisa (2016). Chemical composition of Alhagi sparsifolia flowers, Chem. Nat. Comp. 52(6), 1095-1097.

[10] L. Y. Xiong, H. J. Song, L. Lin and C. Zhang (2015). Chemical constituents of Miscanthus floridulus, Chem. Nat. Comp. 51(3), 552-553.

[11] Y. J. Chen, H. T. Wang, L. P. He, H. G. Zhang and J. L. Yin (2009). Study on chemical constituents from bark of Fraxinus mandschurica Rupr, Chin. Pharm. J. 44(15), 1133-1136.

[12] Y. Kikuchi, Y. Miyaichi, Y. Yamaguchi, H. Kizu and T. Tomimori (1991). Studies on the nepalese crude drugs. XII. On the phenolic compounds from the root of Scutellaria prostrata Jacq. ex Benth, Chem. Pharm. Bull. 39 , 1047-1050.

[13] R. Y. Yang, Y. S. Lan, Z. J. Huang, C. L. Shao, H. Liang, Z. F. Chen and J. Li (2012). Isoflavonoids from Sophora tonkinensis, Chem. Nat. Comp. 48, 674-676.

[14] G. V. Rao, B. N. Swamy, V. Chandregowda and G. C. Reddy (2009). Synthesis of ( \pm )abyssinone I and related compounds: their anti-oxidant and cytotoxic activities, Eur. J. Med. Chem. 44, 2239-2245.

[15] S. Sato, J. Takeo, C. Aoyama and H. Kawahara (2007). $\mathrm{Na}^{+}$-Glucose cotransporter (SGLT) inhibitory flavonoids from the roots of Sophora flavescens, Bioorg. Med. Chem. 15, 3445-3449.

[16] N.M. Mostafa (2018). $\beta$-amyrin rich Bombax ceiba leaf extract with potential neuroprotective activity against scopolamine-induced memory impairment in rats, Rec.Nat.Prod. 12(5), 480-492.

[17] S. K. Sharma, N. Vasudeva and M. Ali (2009). A new aliphatic acid from Achyranthes aspera Linn. roots, Indian J. Chem. 48B, 1164-1169.

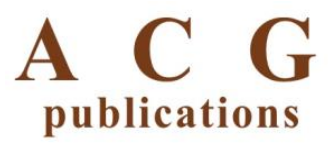

(C) 2019 ACG Publications 